\title{
Breast Cancer Incidence and Behavior in Younger Patients: A Study From the Surveillance, Epidemiology and End Results Database
}

\author{
Zuha Nasimª, Christine Girtain ${ }^{\mathrm{a}}$, Varsha Gupta ${ }^{\mathrm{b}}$, Ishan Patel ${ }^{\mathrm{b}}$, \\ Mohammad A. Hossain ${ }^{\mathrm{b}, \mathrm{c}, \mathrm{d}}$
}

\begin{abstract}
Background: Breast cancer screening for women less than 40 years old is practically non-existent. Since screening can detect cancer at an early stage, not having a surveillance guideline for breast cancer in younger women can result in detection of the cancer at advanced stages. The purpose of this study was to investigate the incidence and behavior of breast cancer in younger women.
\end{abstract}

Methods: The Surveillance, Epidemiology and End Results (SEER) registry data from 2004 to 2014 were accessed for the study. All women diagnosed with breast cancer and with complete information were included in the study. The data were further divided into two groups based on the age of the patient at the time of diagnosis. The younger group consisted of women $<40$ years old (group 1) and the older group consisted of women $\geq 40$ years old (group 2). Both groups were compared on demography and characteristics of the cancer. The continuous variables were tested using Student's $t$-tests and categorical variables were compared using Chi-squared or Fisher's exact tests. Multivariate analysis was done to find the association of high-grade cancer using a logistic regression model. All P-values are two-sided and values $<0.05$ were considered statistically significant.

Results: Of 599,782 patients who satisfied the inclusion criteria, 28,243 (4.71\%) diagnosed with breast cancer were younger women aged $<40$ years old. A higher proportion of these younger women presented with larger tumor sizes (between 5.1 and $10.0 \mathrm{~cm})(11.61 \%$ vs. $5.70 \%, \mathrm{P}<0.001)$, poorly differentiated cancer cells $(55.88 \%$ vs. $32.85 \%, \mathrm{P}<0.001)$ and triple negative receptors $(6.83 \%$ vs. $3.81 \%, \mathrm{P}$ $<0.001)$ than older women respectively. Younger age was significantly associated with high-grade tumor at presentation when controlling

Manuscript submitted March 11, 2020, accepted May 6, 2020

aToms River High School North, Toms River, NJ, USA

${ }^{b}$ Department of Medicine, Jersey Shore University Medical Center, Hackensack Meridian Health, Neptune, NJ 07753, USA

'Hackensack Meridian School of Medicine at Seton Hall University, Nutley, NJ, USA

${ }^{\mathrm{d}}$ Corresponding Author: Mohammad A. Hossain, Hackensack Meridian School of Medicine at Seton Hall University, Nutley, NJ, USA.

Email: Mohammad.Hossain@HackensackMeridian.org

doi: https://doi.org/10.14740/wjon1278 for race and marital status. There was roughly 3\% increased risk of a high-grade tumor with each decrease of 1 year (odds ratio $0.97,95 \%$ confidence interval $0.96-0.99, \mathrm{P}=0.001$ ).

Conclusion: This study found that the proportion of breast cancer cases in younger women was just below 5\%; however, when the cancer was diagnosed, these women presented in advanced stages and more aggressive cancer types.

Keywords: Breast cancer; Young age; Prognosis

\section{Introduction}

Breast cancer is the most common type of cancer in women, excluding a few minor skin cancers [1]. The 2013 statistics show that the lifetime probability and the risk of developing breast cancer is $12.29 \%$, or one in every eight women [2]. Centers for Disease Control and Prevention (CDC) data from 2014 in the United States showed 239,109 cases of breast cancer diagnoses within the year [1], while in 2012, data showed 1.7 million diagnoses of breast cancer worldwide [3]. Breast cancer specifically is the most frequently diagnosed cancer and the leading cause of cancer death among women; female breast cancer accounts for $23 \%$ of total cancer cases and $14 \%$ of deaths by cancer [3]. In 2000, the resulting mortality of the cancer included roughly 375,000 deaths [4].

Breast cancer develops most commonly in women that are 50 years of age or older [5]. A majority of studies are conducted with sample subjects from 50 years and older to study breast cancer overall [6]. Women between the ages of 50 and 74 are recommended to get screening mammograms every 2 years [7], so that the cancer can be detected at an early stage. Early intervention resulted in a decrease in the mortality rate among the older age group of women [8]. There are far less cases of breast cancer in women under 40 . The percent of possible development of breast cancer at the age of 30 is $0.44 \%$, or 1 in 228 , which is lower than the percentage of any older age group [4]. Breast cancer does not follow more commonly observed linear relationships between incidence and age [9], suggesting a complex role of various risk factors in causing early development. Risk factors for developing cancer at an earlier age may include 
both internal and external hormonal factors: age at first menarche, age at menopause, age of first pregnancy [10]. Breast cancer in younger women can possibly be observed, on average, as diagnosed at further advanced stages due to factors such as delayed presentation, unfavorable tumor characteristics, genetic mutations, and/or family history of breast cancer [11].

Since there is not a clear guidance in the screening of early detection of breast cancer in younger age group women compared to established guidelines for the older women, the study was designed to look at the incidence and behavior of breast cancer at the time of the diagnosis in younger women.

\section{Hypothesis}

Breast cancer in younger women $(<40$ years old $)$ will be diagnosed at a higher grade than for older women $(\geq 40)$.

\section{Materials and Methods}

\section{Data source}

Cases for breast cancer patients were collected from the public National Cancer Institute (NCI) Surveillance, Epidemiology and End Results (SEER) Program database. Collaborative Institutional Training Initiative (CITI Program) Training was completed for access to the SEER data, which was provided for the principal investigator by a biostatistician from Jersey Shore University Medical Center. The database provides access to demography, tumor behavior and histology. The data are de-identified and anonymous, with the database being the largest repository of cancer cases in the United States. The SEER registries house millions of cases of cancer patients all across the United States: San Francisco-Oakland, Connecticut, Metropolitan Detroit, Hawaii, Iowa, New Mexico, Seattle, Utah, Metropolitan Atlanta, Alaska, San Jose-Monterey, Los Angeles, Rural Georgia, Greater California, Kentucky, Louisiana, New Jersey and Greater Georgia.

\section{Inclusion and exclusion criteria}

Cases were extracted from the SEER database for the sampling time frame between 2004 and 2014. All female patients who were diagnosed with breast cancer and confirmed with histology and/or cytology were included in the study. The patient's demographic characteristics including age, race and marital status at the time of diagnosis were collected. Other variables included in the study were tumor characteristics: size, location, histology, extension, metastasis, receptor status, stage and grades of the tumor (high grade: grades III and IV; low grade: grades I and II). All male breast cancer cases were excluded from the study. Breast cancer cases diagnosed at autopsy were also excluded. Variables were excluded from the overall dataset for more accurate analysis, including variables in accordance with old coding systems, variables with too many missing (NA) code values and variables that proved irrelevant to the purpose of the study. Listwise deletion was then performed for the remaining variables to delete values that were unknown, inapplicable, or unspecified. These values were set as missing values and further deleted to create a clean dataset. Any variables with only missing values that remained from the selection process were removed from the dataset to prevent data corruption. Duplicate cases were then dropped from the dataset to prevent inaccurate analysis (Fig. 1).

\section{Statistical analysis}

The data set was divided into a younger age group (age $<40$ years old) and older age group ( $\geq 40$ years old). The two groups were compared on patients' characteristics and tumor characteristics. The summary statistics were obtained and presented the following values for both age groups for the continuous variables: mean, standard deviation (SD), median and interquartile range (IQR; first and third quartiles). The Student's $t$-test was used for each continuous variable. The categorical variables were compared between the two groups using Pearson's Chi-squared $\left(\chi^{2}\right)$ test or Fisher's exact test. In order to eliminate confounding variables, multivariate logistic regression analysis was performed to examine relationships between high-grade tumors and age of the patient at the time of diagnosis. The statistical coding language $\mathrm{R}$ was used with Rstudio, its integrated development environment (IDE), as well as packages "dplyr", "stats" and "psych" within the IDE for efficiency in data manipulation and statistical functionality when analyzing the data. All P-values are two-sided and the values $<$ 0.05 are considered as statistically significant.

The primary outcome of the study was the stage of the tumor at the time of diagnosis. Secondary outcomes were tumor receptors' status and presence of metastasis.

\section{Results}

A total of 810,835 cases were reported in the SEER registry from 2004 to 2014. Out of those, 599,782 cases satisfied the inclusion criteria. A majority, 571,539 (95.29\%) of patients who were diagnosed with breast cancer were 40 years old or above (older group) and the remaining 28,243 (4.71\%) belonged to the younger group. The mean (SD) age of the younger group was 34.89 (3.80) years and the mean (SD) age of the older group was $62.158(12.51)$. When comparing the marital status, a higher proportion of patients in the younger group were single compared to the older group $(28.80 \%$ versus $13.39 \%$, $\mathrm{P}<0.001)$. Regarding race, there was a higher percentage of black women with breast cancer cases identified in the younger group compared to the older group $(15.32 \%$ versus $10.26 \%, \mathrm{P}$ $<0.001$ ). The most common primary site (the site that cancer originated) between both groups was the upper-outer quadrant of the breast, split almost evenly between cases involving either the right or left breast, with almost all cases having diagnoses confirmed with positive histology (Table 1).

When comparing the outcomes between the two groups, younger group women displayed higher percentages of tumors 


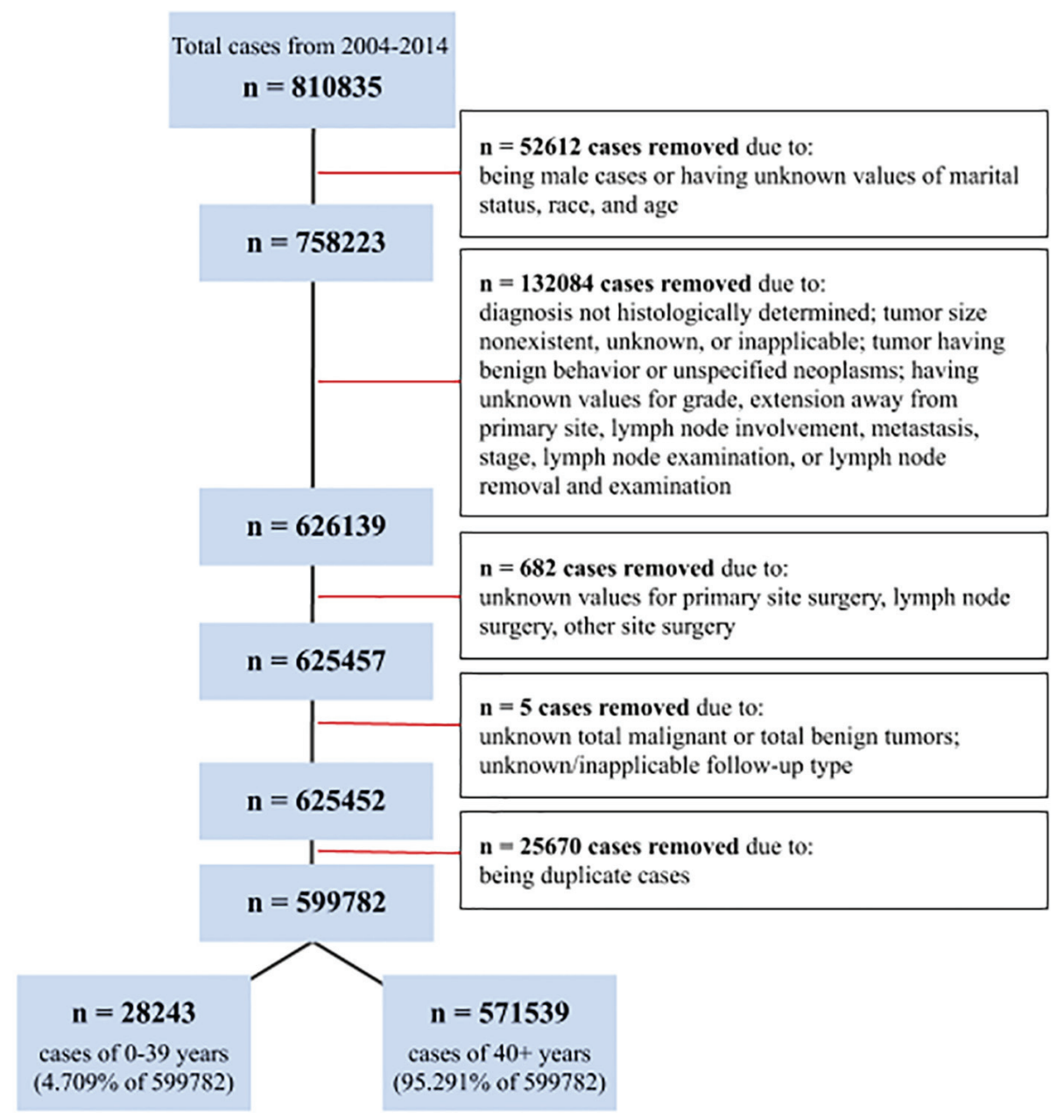

Figure 1. Inclusion and Exclusion Criteria.

in size ranges $2.1-5.0 \mathrm{~cm}$ and $5.1-10.0 \mathrm{~cm}$ when compared to the older group $(42.65 \%$ vs. $28.73 \%$ and $11.61 \%$ vs. $5.70 \%$, P $<0.001$ ), respectively (Table 2 ). A higher percentage of cases from the younger group had regional as well as distant metastasis when compared to older group (40.76\% vs. $24.93 \%$ and $6.83 \%$ vs. $3.81 \%, \mathrm{P}<0.001)$. Similarly, the younger group also had higher percentages of poorly differentiated cancerous cells when compared to the older group $(55.88 \%$ vs. $32.85 \%, \mathrm{P}<$ $0.001)$. Younger women displayed a higher percentage of invasive carcinoma at their primary site compared to older women (91.45\% vs. $85.23 \%, \mathrm{P}<0.001)$. Regarding receptor assay analysis, estrogen receptor (ER) assay, progesterone receptor (PR) assay and human epidermal growth factor receptor 2 (HER2) protein analysis, triple negative receptors assay ER(-), PR(-) and HER2(-) cases were found to be almost double in the younger group compared to the older group $(7.83 \%$ vs. $4.31 \%$, $\mathrm{P}<0.01$ ) (Table 2). Analysis of stage based on TNM classification (AJCC seventh edition) displayed younger women having approximately double the percentage of stage 4 cancers compared to older women $(2.42 \%$ vs. $1.48, \mathrm{P}<0.001)$ (Table 3$)$.

When the high-grade tumor was assessed in the multivariate logistic regression analysis controlling for race and mari- tal status, the younger age group was significantly associated with a higher percentage of high-grade tumor development. A decrease of 1 year of age related to almost 3\% increased risk of a high-grade tumor (odds ratio (OR) $0.97,95 \%$ confidence interval (CI) $0.96-0.99, \mathrm{P}=0.001$ ) (Table 4).

When the age was further analyzed through multivariate logistic regression to determine the correlation between the age group and the grading of the tumor, it was found that the younger age group has 2.437 higher odds (95\% CI 2.38 - 2.50, $\mathrm{P}<0.001)$ of having a high-grade tumor at the time of diagnosis (Table 5).

\section{Discussion}

From the 599,782 SEER cases analyzed in this study, 28,243 cases were diagnosed in women 0 - 39 years of age, making up $4.71 \%$ of the total. Evidence demonstrates these younger women being diagnosed with more aggressive tumors and cancerous growths compared to women diagnosed at 40 years of age and older. The qualitative aggressiveness is based upon the younger group having a higher proportion of high-grade diag- 


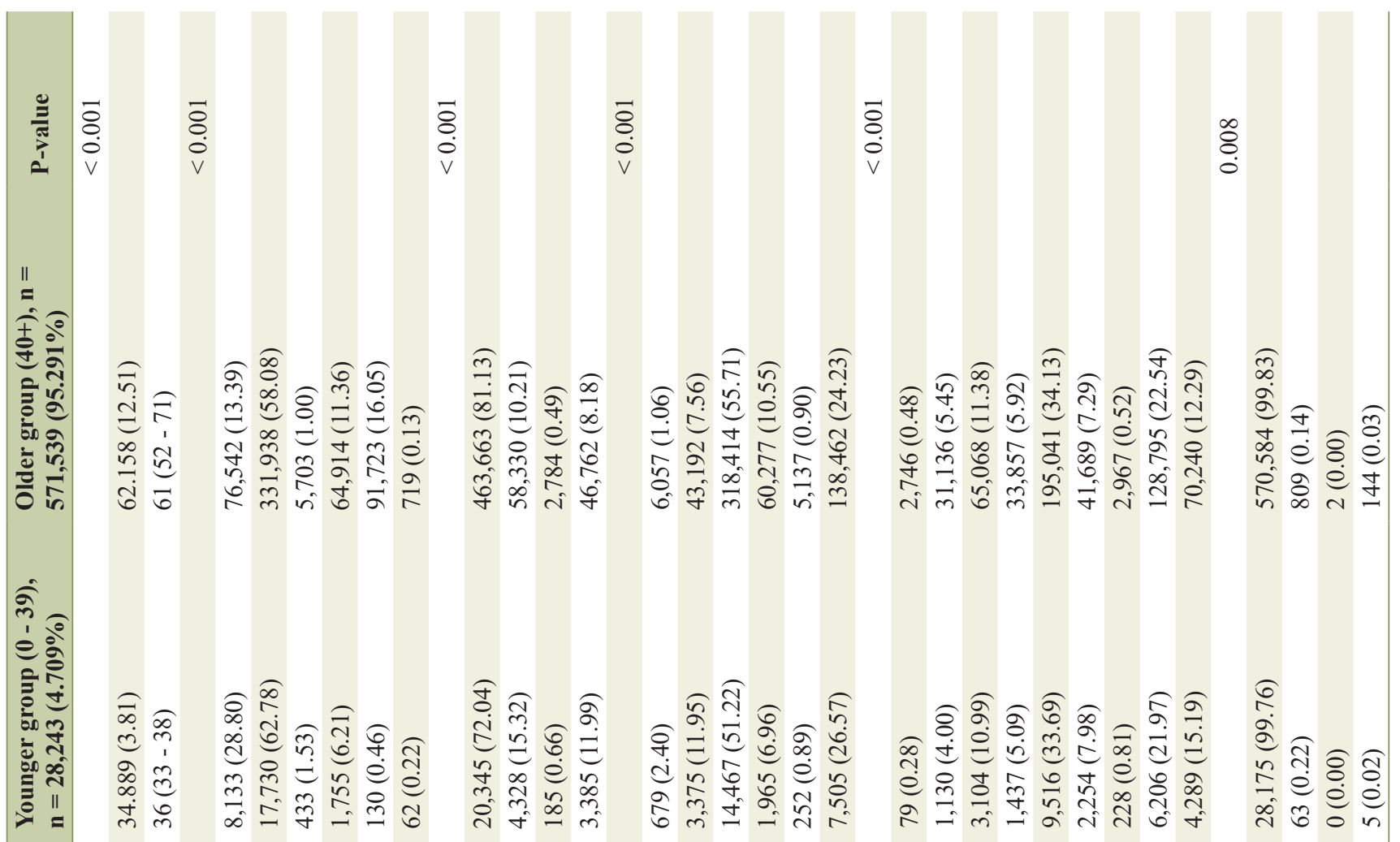

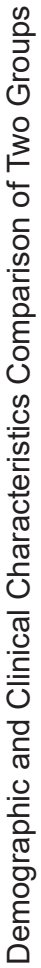

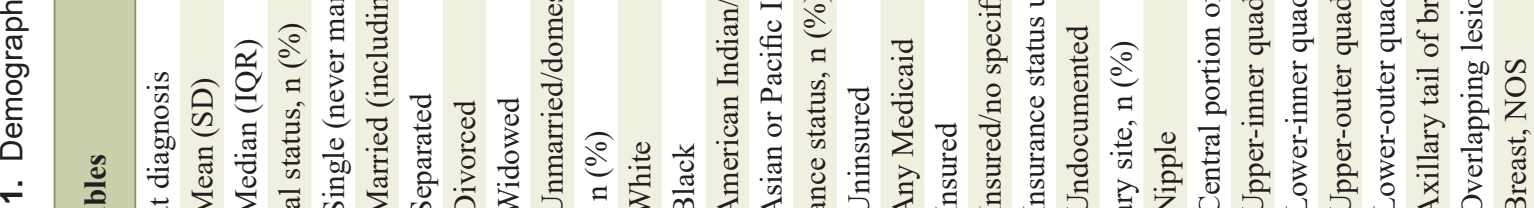

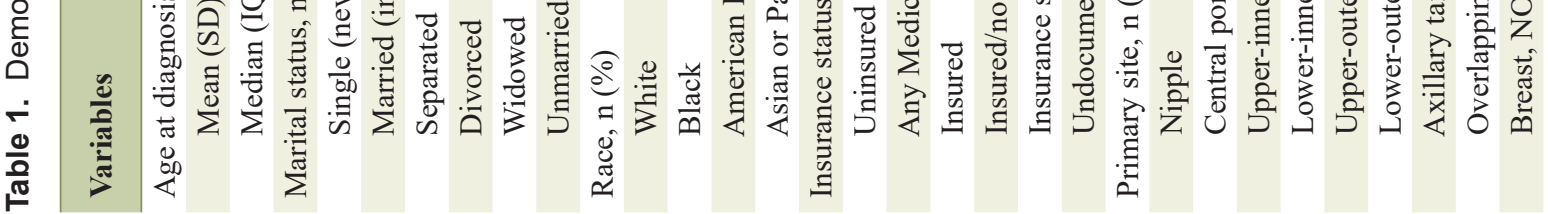




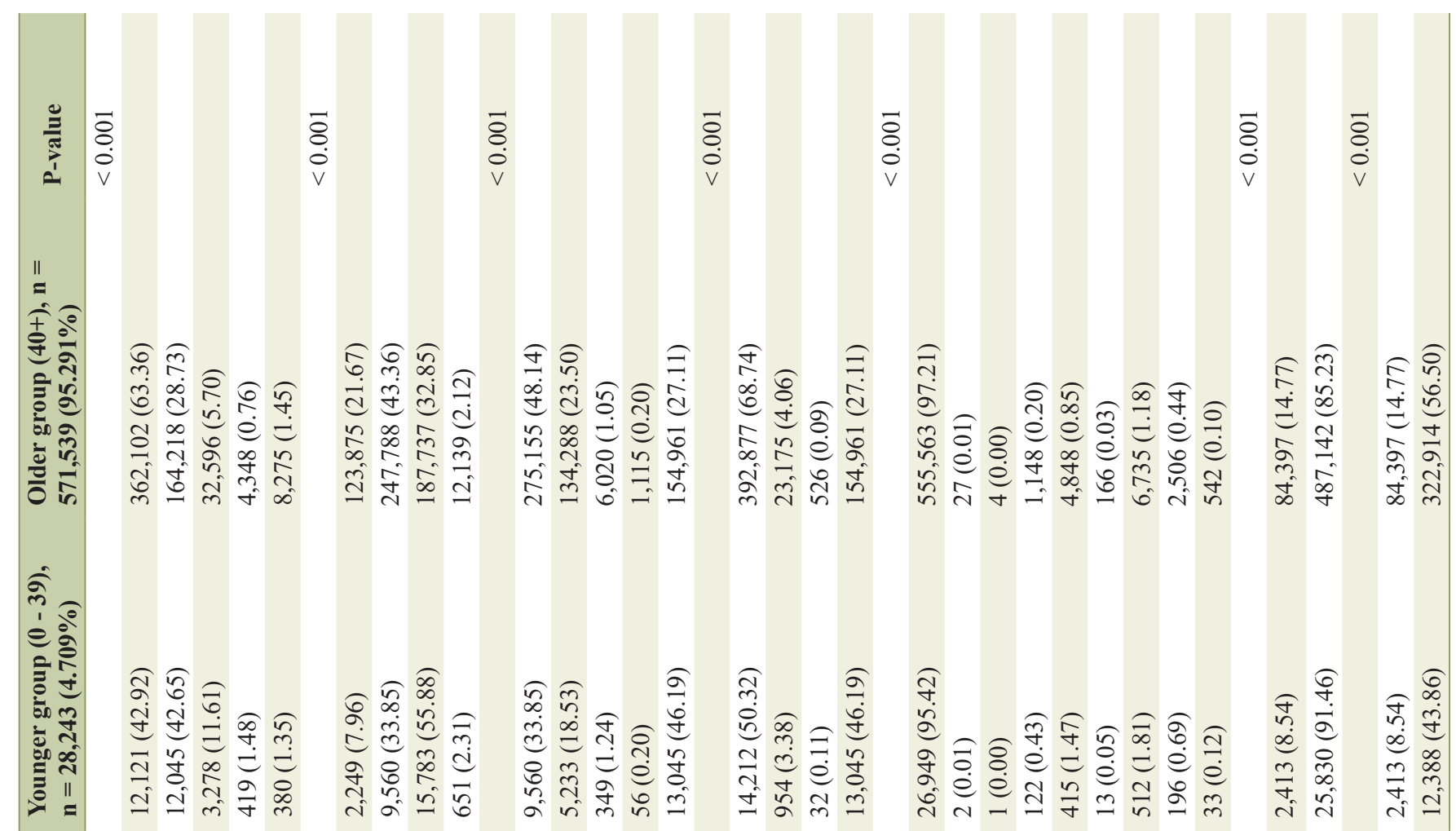

0
3
0
0
0
0
0
0
0
0
0
0
0
3
0
0
0
0
0
0
0
0
0
0
0
0
0
0
0
5
5
1

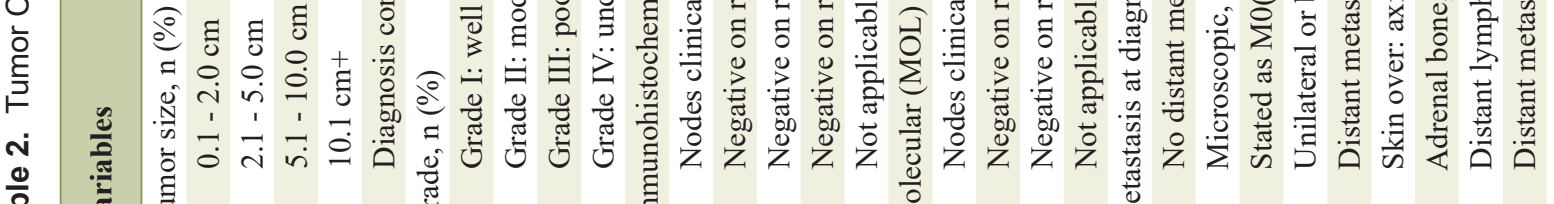

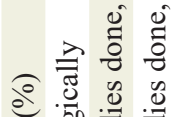

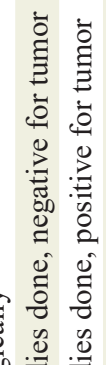

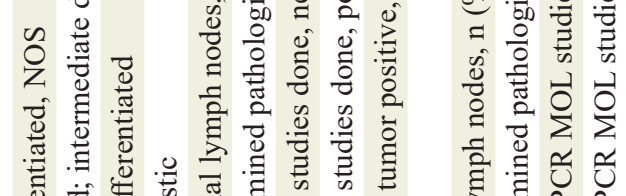
की

=

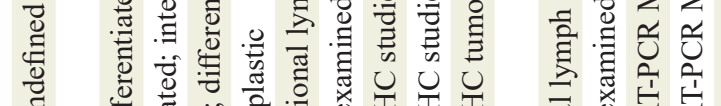

范苍离

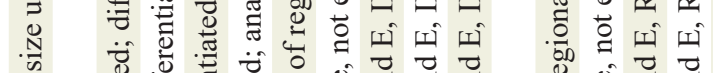

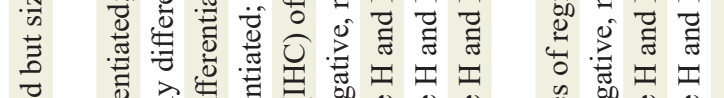

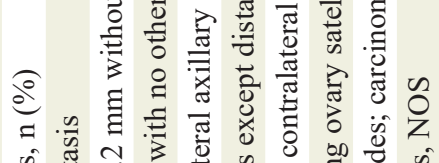

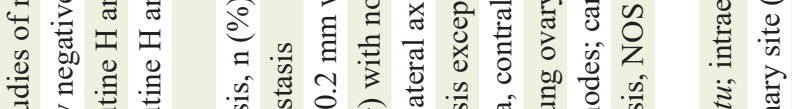




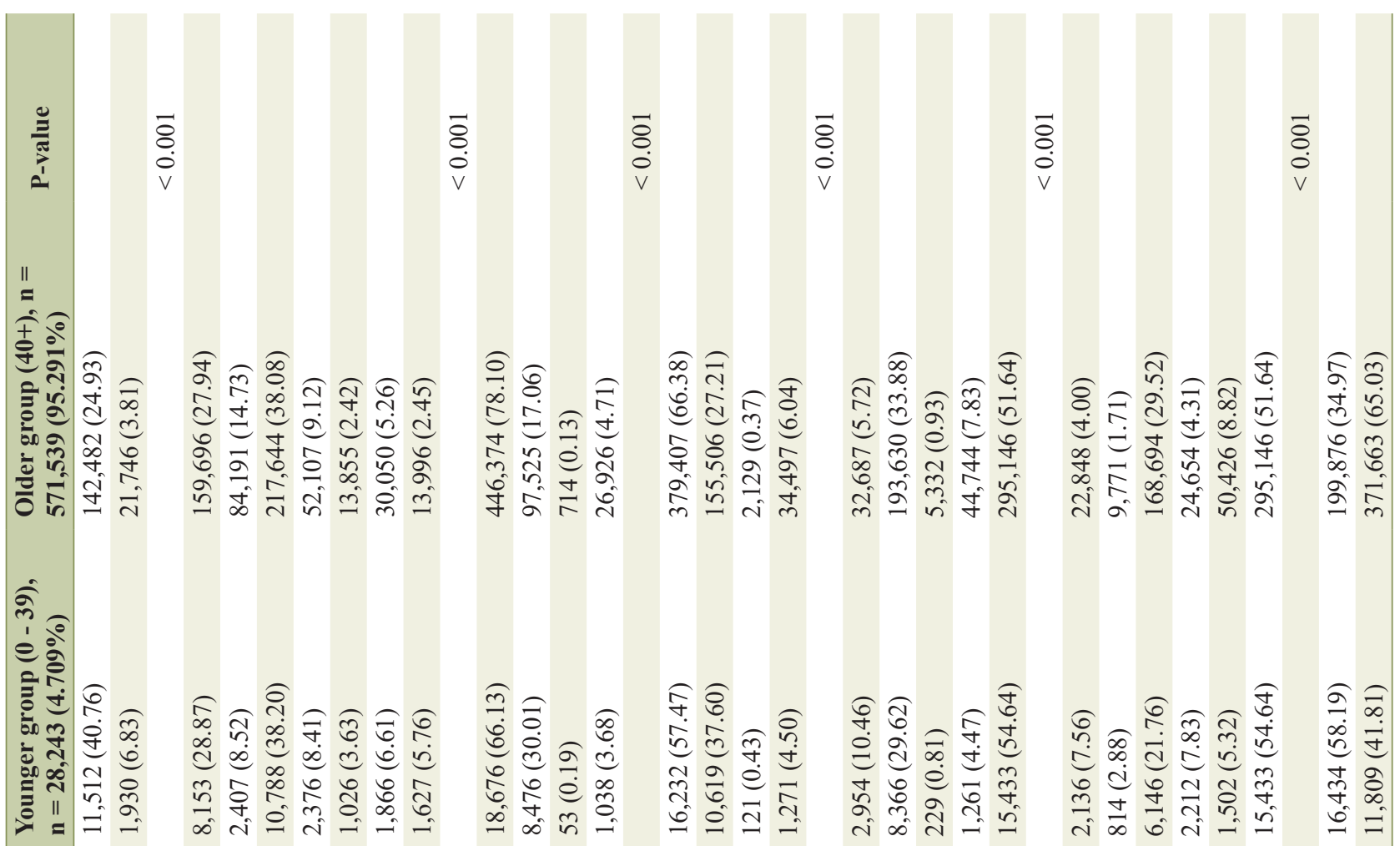

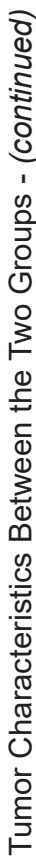
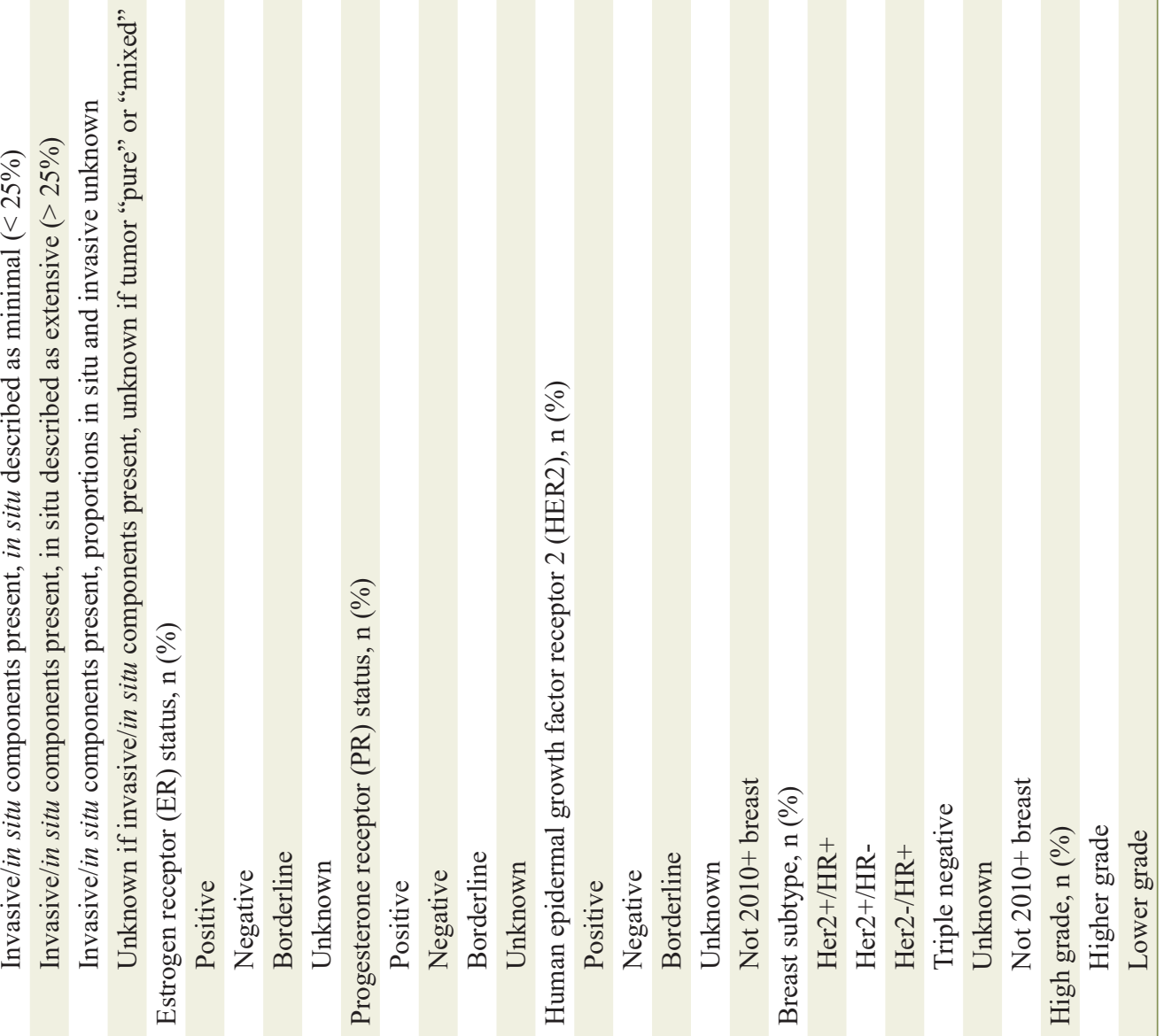
Table 3. Adjusted Stage Based on TNM Classification (Seventh Edition)

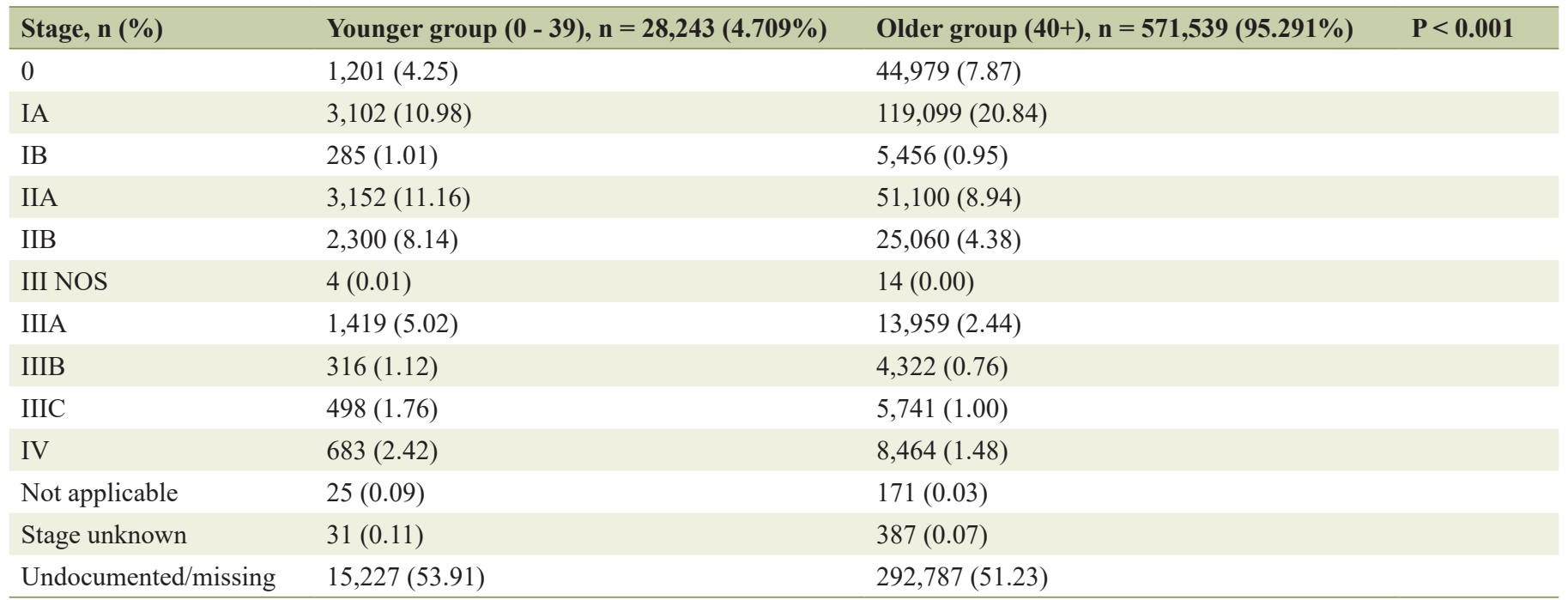

NOS: not otherwise specified.

noses, as well as higher invasiveness, more distant metastases, the increased likeliness of carcinomatosis and larger tumors.

Incidence of breast cancer in younger women is lower than in older women, increasing as age increases. However, women at risk under 40, under the recommended screening age, can be more prone to aggressive tumors, putting them at higher risk of fatality [12]. A study examining cases from 1989 to 2009 in Galway University Hospital displayed similar results on a smaller scale. The study showed younger women to have significantly higher grade and stage, as well as higher HER2 over-expression [11]. A San Antonio database study revealed younger women to have the worst survival outcome when compared to older women at the same stages of cancer. Analyses showed more positive lymph nodes, larger tumors and negative steroid hormone receptors in younger patients [13]. Younger women with the same advanced stages of breast cancer as older women demonstrated breast cancer to be more fatal. An additional study examining 72,367 SEER database cases from 1973 to 1998 displayed younger women with distant metastasis to have poorer prognosis and a higher likelihood of fatality [14].

When examining breast cancer cases of women in Asia, a study found that women below 35 presented poorer prognostic profiles, including higher-grade tumors and more prevalent nodal involvement [15]. A Singapore-Malaysia hospital database study found that women younger than 50 comprised $51 \%$ of diagnosed cases, roughly double the percent under 50 years old in the USA [16]. An additional study of Asian Indian/Pakistani women in the USA from the SEER database presented this group of women to demonstrate more ER/PR negative cases than Caucasians. This group of Indian/Pakistani women also had roughly $10 \%$ more cases of women diagnosed at ages less than 40 compared to Caucasians. However, survival outcome

Table 4. Multivariate Analysis of High-Grade Tumor Using Age Group

\begin{tabular}{llll}
\hline Covariate & Odds ratio & P-value & 95\% confidence interval \\
\hline Race & & & \\
$\quad$ Black & 1.660 & $<0.001$ & $1.63-1.69$ \\
$\quad$ American Indian/Alaska Native & 1.138 & 0.001 & $1.06-1.23$ \\
$\quad$ Asian or Pacific Islander & 1.074 & $<0.001$ & $1.05-1.10$ \\
Marital status & & & $0.96-0.99$ \\
$\quad$ Married (including common law) & 0.973 & 0.001 & $1.00-1.12$ \\
$\quad$ Separated & 1.057 & 0.040 & $0.99-1.03$ \\
$\quad$ Divorced & 1.008 & 0.483 & $1.09-1.13$ \\
$\quad$ Widowed & 1.109 & $<0.001$ & $0.73-0.99$ \\
$\quad$ Unmarried/domestic partner & 0.853 & 0.036 & $0.98-0.98$ \\
Age at diagnosis & 0.978 & $<0.001$ & $1.97-2.09$ \\
Constant & 2.025 & $<0.001$ & \\
\hline
\end{tabular}

White race and single marital status were used as a reference in the analysis. 
Table 5. Multivariate Analysis of Age Group With High-Grade Tumor

\begin{tabular}{llll}
\hline Covariate & Odds ratio & P-value & 95\% confidence interval \\
\hline $\begin{array}{l}\text { Younger vs older age } \\
\text { Marital status }\end{array}$ & 2.437 & $<0.001$ & $2.38-2.50$ \\
$\quad$ Married (including common law) & 0.535 & $<0.001$ & $0.52-0.55$ \\
$\quad$ Separated & 0.696 & $<0.001$ & $0.63-0.77$ \\
$\quad$ Divorced & 0.267 & $<0.001$ & $0.25-0.28$ \\
$\quad$ Widowed & 0.015 & $<0.001$ & $0.01-0.02$ \\
$\quad$ Unmarried or domestic partner & 0.898 & 0.422 & $0.69-1.17$ \\
Race & & & $1.27-1.37$ \\
$\quad$ Black & 1.318 & $<0.001$ & $1.16-1.57$ \\
$\quad$ American Indian/Alaska Native & 1.347 & $<0.001$ & $1.44-1.55$ \\
$\quad$ Asian or Pacific Islander & 1.491 & $<0.001$ & $0.06-0.06$ \\
Constant & 0.062 & $<0.001$ & \\
\hline
\end{tabular}

High grade: grades III and IV. Low grade (grades I and II), white race and single marital status were used as references in the analysis.

was generally similar to that of Caucasians, while that of African American women was worse [17]. Considering African American females showed a higher percentage of breast cancer cases identified in the younger group, a study focusing on African-British women showed black women presenting 21 years younger than white women on average [18]. This suggests an increased risk for this race group in America and Britain.

The most common primary site of breast cancer being the upper-outer quadrant of the breast may be correlated with the greater amount of breast tissue and density in the specific region of the breast [19]. Although this quadrant of the breast is closest to the axillary lymph nodes and cancer may spread to these nodes over time, the original tumor location is not an independent prognostic factor in determining metastasis [20].

There are numerous reports which demonstrate that the major advances in survival documented in young breast cancer patients in the last decade have resulted from advances in treatment as these improvements in overall survival (OS) are documented in screened and unscreened populations. Pathologic complete response to neoadjuvant systemic therapy in high-risk cancers such as triple negative and Her-2 driven cancers appear to be independent of stage I-III, and thus it is likely that the biological behavior of these tumors is dictated by chemosensitivity more than by their stage. Thus, Guo et al [21] in an excellent SEER-based study of breast cancer in young women concluded that screening has contributed little to the improvements in OS in this population. They concluded that even in the screened population of women $>40$ "nearly all of the mortality reduction was caused by treatment advances and NOT screening".

In a similar vein, Anders et al [22] concluded that the increased proportion of aggressive intrinsic tumor subtypes (most of which are high-grade lesions) can account for differences in tumor behavior in young women. They suggested that new targeted chemotherapy and systemic therapy can erase the importance of age as a prognostic factor.

Younger women that develop breast cancer often suffer prolonged psychological and physical issues including menopause-related concerns, weight gain and physical inactivity
[23]. This raises concerns to diagnose breast cancer in younger women at earlier stages to decrease prolonged issues and improve survival outcomes.

This study utilizes one of the largest sample sizes, 599,782 cases, analyzed from the SEER database. The age-based analysis of outcome factors indicated younger women to have higher likeliness of being diagnosed at advanced stages of breast cancer as opposed to older women who are more regularly screened. The larger growth may possibly result from a lack of screening guidelines concerning younger women. However, regular screening by digital mammography in women aged 40 - 49 years showed lower specificity. Therefore, the current recommendation for screening in this age group is to individualize patients based on risk factors [24]. Self-breast examinations and physician examinations yielded even lower positive results in women younger than 40 years old $[25,26]$. As studies involving women below 40 are scarcer, there is less understanding on how to narrow screenings for the likelihood of developing cancer for these individuals. This study validates the results of other studies that younger women with breast cancer are diagnosed with advanced stages of cancer compared to older women $[11,12,14,27]$. The higher mortality among younger patients who presented with the same stage breast cancer as older women [13] provides an insight and need to create some strategies to identify cancer at an early stage. Despite the mortality rate plateauing in breast cancer in women between the ages of 20 and 29 years since 2005, the incidence of metastatic disease in this age group continues to increase [26]. Removal of acquired risk factors, early detection and timely interventions should be the few steps towards tackling a very difficult problem younger women face.

\section{Limitations}

The study was performed utilizing retrospective data from the SEER database, constituting inherent bias in the study sample. 
As the cases in the database were de-identified with a set guideline of variables, detailed information regarding individual hormonal factors was not included: age of first menarche, age of first pregnancy, age at menopause. Since no such information was available, consideration of these factors was left out of the study design. As a result of analyzing the database, certain variables were excluded based on consideration of the sample size as well as a lack of information regarding different variables.

\section{Conclusion}

Breast cancer is indicated to be diagnosed as more aggressive at higher grades in younger women when compared to diagnoses in older women. Tumors of these cases are often observed to be more invasive at the primary site while having more regional and distant metastases. Additionally, younger cases display larger tumors with more characteristics of poor prognosis.

\section{Future work}

A future study may be conducted with the database that can provide detailed information of the characteristics of younger women. Further studies may also be conducted focusing more on risk for groups of women from different countries living in the USA. This may provide more evidence of risk for specific groups of women that could further narrow developing screening guidelines.

\section{Acknowledgments}

We acknowledge Nasim Ahmed for downloading de-identified data from the SEER database.

\section{Financial Disclosure}

This project was not supported by any grant or funding agencies.

\section{Conflict of Interest}

The authors declare that there is no conflict of interest regarding the publication of this paper.

\section{Informed Consent}

Not applicable.

\section{Author Contributions}

Each author has individually been involved in and has made substantial contributions to conception and design, acquisition of data, analysis, interpretation of data, drafting and editing the manuscript. Zuha Nasim: conceptualization and methodology, data analysis and data collection, statistical analysis and data interpretation, drafting the manuscript. Christine Girtain: data interpretation and manuscript revision. Varsha Gupta and Ishan Patel: manuscript revision and approval. Mohammad A. Hossain: data interpretation, manuscript revision and final approval.

\section{Data Availability}

The authors declare that data supporting the findings of this study are available within the article.

\section{References}

1. Centers for Disease Control and Prevention (CDC). 2018. Breast Cancer Statistics. Retrieved from https://www.cdc. gov/cancer/breast/statistics/index.htm.

2. DeSantis C, Ma J, Bryan L, Jemal A. Breast cancer statistics, 2013. CA Cancer J Clin. 2014;64(1):52-62.

3. Jemal A, Bray F, Center MM, Ferlay J, Ward E, Forman D. Global cancer statistics. CA Cancer J Clin. 2011;61(2):69-90.

4. Bray F, McCarron P, Parkin DM. The changing global patterns of female breast cancer incidence and mortality. Breast Cancer Res. 2004;6(6):229-239.

5. Centers for Disease Control and Prevention (CDC). What Are the Risk Factors for Breast Cancer? 2018. Retrieved from https://www.cdc.gov/cancer/breast/basic_info/risk_ factors.htm.

6. Trends in incidence of breast cancer among women under 40 in seven European countries: A GRELL cooperative study. 2013. Retrieved from https://www.sciencedirect. com/science/article/pii/S1877782113000775.

7. Centers for Disease Control and Prevention (CDC). What is a mammogram? 2018. Retrieved from https://www. cdc.gov/cancer/breast/basic_info/mammogram.htm.

8. Howe HL. Repeat Mammography among women over 50 years of age. 1992. Retrieved from https:/www.ajpmonline.org/article/S0749-3797(18)30829-8/abstract.

9. Pike MC, Krailo MD, Henderson BE, Casagrande JT, Hoel DG. 'Hormonal' risk factors, 'breast tissue age' and the age-incidence of breast cancer. Nature. 1983;303:767770 .

10. Molinie F, Vanier A, Woronoff AS, Guizard AV, Delafosse P, Velten M, Daubisse-Marliac L, et al. Trends in breast cancer incidence and mortality in France 1990-2008. Breast Cancer Res Treat. 2014;147(1):167-175.

11. Kheirelseid EH, Boggs JM, Curran C, Glynn RW, Dooley $\mathrm{C}$, Sweeney KJ, Kerin MJ. Younger age as a prognostic indicator in breast cancer: a cohort study. BMC Cancer. 2011;11:383.

12. Yankaskas BC. Epidemiology of breast cancer in young women. Breast Dis. 2005;23:3-8.

13. Albain KS, Allred DC, Clark GM. Breast cancer outcome 
and predictors of outcome: are there age differentials? J Natl Cancer Inst Monogr. 1994;16:35-42.

14. Rosenberg J, Chia YL, Plevritis S. The effect of age, race, tumor size, tumor grade, and disease stage on invasive ductal breast cancer survival in the U.S. SEER database. Breast Cancer Res Treat. 2005;89(1):47-54.

15. Foo CS, Su D, Chong CK, Chng HC, Tay KH, Low SC, Tan SM. Breast cancer in young Asian women: study on survival. ANZ J Surg. 2005;75(7):566-572.

16. Pathy NB, Yip CH, Taib NA, Hartman M, Saxena N, Iau P, Bulgiba AM, et al. Breast cancer in a multi-ethnic Asian setting: results from the Singapore-Malaysia hospital-based breast cancer registry. Breast. 2011;20(Suppl 2):S75-80.

17. Kakarala M, Rozek L, Cote M, Liyanage S, Brenner DE. Breast cancer histology and receptor status characterization in Asian Indian and Pakistani women in the U.S.- - a SEER analysis. BMC Cancer. 2010;10:191.

18. Bowen RL, Duffy SW, Ryan DA, Hart IR, Jones JL. Early onset of breast cancer in a group of British black women. Br J Cancer. 2008;98(2):277-281.

19. Lee AH. Why is carcinoma of the breast more frequent in the upper outer quadrant? A case series based on needle core biopsy diagnoses. Breast. 2005;14(2):151-152.

20. Rummel S, Hueman MT, Costantino N, Shriver CD, Ellsworth RE. Tumour location within the breast: Does tumour site have prognostic ability? Ecancermedicalscience. 2015;9:552.

21. Guo F, Kuo YF, Shih YCT, Giordano SH, Berenson AB. Trends in breast cancer mortality by stage at diagnosis among young women in the United States. Cancer. 2018;124(17):3500-3509.

22. Anders CK, Fan C, Parker JS, Carey LA, Blackwell KL, Klauber-DeMore N, Perou CM. Breast carcinomas arising at a young age: unique biology or a surrogate for aggressive intrinsic subtypes? J Clin Oncol. 2011;29(1):e18-20.

23. Howard-Anderson J, Ganz PA, Bower JE, Stanton AL. Quality of life, fertility concerns, and behavioral health outcomes in younger breast cancer survivors: a systematic review. J Natl Cancer Inst. 2012;104(5):386-405.

24. Siu AL, Force USPST. Screening for Breast Cancer: U.S. Preventive services task force recommendation statement. Ann Intern Med. 2016;164(4):279-296.

25. Gili AF, Poonja Z, Kalra BB. Breast cancer screening for women younger than 40. Can Fam Physician. 1993;39:6572.

26. Desreux JAC. Breast cancer screening in young women. Eur J Obstet Gynecol Reprod Biol. 2018;230:208-211.

27. Murphy BL, Day CN, Hoskin TL, Habermann EB, Boughey JC. Adolescents and young adults with breast cancer have more aggressive disease and treatment than patients in their forties. Ann Surg Oncol. 2019;26(12):3920-3930. 